TRANSACTIONS OF THE

AMERICAN MATHEMATICAL SOCIETY

Volume 365, Number 11, November 2013, Pages 5911-5932

S 0002-9947(2013)05881-3

Article electronically published on July 2, 2013

\title{
THE BISHOP-PHELPS-BOLLOBÁS THEOREM FOR BILINEAR FORMS
}

\author{
MARÍA D. ACOSTA, JULIO BECERRA-GUERRERO, DOMINGO GARCÍA, \\ AND MANUEL MAESTRE
}

\begin{abstract}
In this paper we provide versions of the Bishop-Phelps-Bollobás Theorem for bilinear forms. Indeed we prove the first positive result of this kind by assuming uniform convexity on the Banach spaces. A characterization of the Banach space $Y$ satisfying a version of the Bishop-Phelps-Bollobás Theorem for bilinear forms on $\ell_{1} \times Y$ is also obtained. As a consequence of this characterization, we obtain positive results for finite-dimensional normed spaces, uniformly smooth spaces, the space $\mathcal{C}(K)$ of continuous functions on a compact Hausdorff topological space $K$ and the space $K(H)$ of compact operators on a Hilbert space $H$. On the other hand, the Bishop-Phelps-Bollobás Theorem for bilinear forms on $\ell_{1} \times L_{1}(\mu)$ fails for any infinite-dimensional $L_{1}(\mu)$, a result that was known only when $L_{1}(\mu)=\ell_{1}$.
\end{abstract}

\section{INTRODUCTION}

E. Bishop and R. Phelps in 4 proved that every continuous linear functional $x^{*}$ on a Banach space $X$ can be approximated, uniformly on the closed unit ball of $X$, by a continuous linear functional $y^{*}$ that attains its norm. This result is called the Bishop-Phelps Theorem. Shortly thereafter, B. Bollobás in [5] showed that this approximation can be done in such a way that, moreover, the point at which $x^{*}$ almost attains its norm is close in norm to a point at which $y^{*}$ attains its norm. This is a "quantitative version" of the Bishop-Phelps Theorem, known as the Bishop-Phelps-Bollobás Theorem. As usual, by $B_{X}$ and $S_{X}$ we will denote the closed unit ball and the unit sphere of a Banach space $X$, respectively, and $X^{*}$ will be the dual of $X$.

Theorem 1.1 (Bishop-Phelps-Bollobás Theorem, [6, Theorem 16.1]). Let $X$ be a Banach space and $0<\varepsilon<1$. Given $x \in B_{X}$ and $x^{*} \in S_{X^{*}}$ with $\left|1-x^{*}(x)\right|<\frac{\varepsilon^{2}}{4}$, there are elements $y \in S_{X}$ and $y^{*} \in S_{X^{*}}$ such that $y^{*}(y)=1,\|y-x\|<\varepsilon$ and $\left\|y^{*}-x^{*}\right\|<\varepsilon$.

In 1 the authors proved versions of the Bishop-Phelps-Bollobás Theorem for operators. Amongst them is shown a characterization of the Banach spaces $Y$

Received by the editors February 6, 2012.

2010 Mathematics Subject Classification. Primary 46B20; Secondary 46B25, 46B28.

Key words and phrases. Bishop-Phelps-Bollobás Theorem, bilinear form, Banach space.

The first author was supported by MICINN and FEDER Project MTM-2009-07498 and Junta de Andalucía "Proyecto de Excelencia" P09-FQM-4911.

The second author was supported by Junta de Andalucía grants FQM 0199 and FQM 1215, and MTM-2011-23843.

The third and fourth authors were supported by MICINN Project MTM2011-22417.

The fourth author was also supported by Prometeo 2008/101 and MEC PR2010-0374.

(C) 2013 American Mathematical Society Reverts to public domain 28 years from publication 
satisfying an analogous result of the Bishop-Phelps-Bollobás Theorem for operators from $\ell_{1}$ into $Y$. There are also positive results for operators from $L_{1}(\mu)$ to $L_{\infty}(\nu)$ [3] and for operators from an Asplund space to $C(K)$ [2. For more results on the subject, also see [7].

Choi and Song initiated the study of versions of the Bishop-Phelps-Bollobás Theorem for bilinear forms [9, showing that this theorem does not hold for $\ell_{1} \times \ell_{1}$. For two Banach spaces $X$ and $Y$, by using the natural identification of the space of the continuous bilinear forms on $X \times Y$ and the space $L\left(X, Y^{*}\right)$ of linear and continuous operators from $X$ into $Y^{*}$, it is clear that the pair $\left(X, Y^{*}\right)$ satisfies the BPBP for operators if the pair $(X, Y)$ has the BPBP for bilinear forms (see the definitions below). The converse is not true even for $X=Y=\ell_{1}$ (see [9] and [1, Theorem 4.1]).

Our aim in this paper is to provide classes of spaces satisfying a version of the Bishop-Phelps-Bollobás Theorem for bilinear forms. Before describing the results that we obtained we will introduce some notation and definitions in order to be more precise.

All the Banach spaces considered in this paper will be over the scalar field $\mathbb{K}$ $(\mathbb{R}$ or $\mathbb{C})$. Except when explicitly stated, all results in this paper hold for the real and the complex cases. If $A$ is a subset of a linear space, we will denote by $\operatorname{co} A$ and $|\mathrm{co}| A$ the convex hull and the absolutely convex hull of $A$, respectively. For a family of Banach spaces $X_{1}, \ldots, X_{n}, Y$ we denote by $L^{n}\left(X_{1} \times \cdots \times X_{n}, Y\right)$ the Banach space of all continuous $n$-linear mappings from $X_{1} \times \cdots \times X_{n}$ to $Y$. When $Y$ is the scalar field we remove it, i.e. we write $L^{n}\left(X_{1} \times \cdots \times X_{n}\right)$. If $n=1$ we simply write $L(X, Y)$ and $X^{*}$ when $Y$ is the scalar field.

In [1] the following property was introduced to study versions of the BishopPhelps-Bollobás Theorem for operators. To deal with the bilinear case we need a natural modification of this property (see Definition 1.3 below).

Definition 1.2 ([1, Definition 1.1]). If $X$ and $Y$ are Banach spaces, the pair $(X, Y)$ satisfies the Bishop-Phelps-Bollobás property for operators (for short, BPBP for operators) if given $\varepsilon>0$, there are $\eta(\varepsilon)>0$ and $\beta(\varepsilon)>0$ with $\lim _{t \rightarrow 0} \beta(t)=0$ such that for all $T \in S_{L(X, Y)}$, if $x_{0} \in S_{X}$ is such that $\left\|T x_{0}\right\|>1-\eta(\varepsilon)$, then there exist a point $u_{0} \in S_{X}$ and an operator $S \in S_{L(X, Y)}$ satisfying the following conditions:

$$
\left\|S\left(u_{0}\right)\right\|=1, \quad\left\|u_{0}-x_{0}\right\|<\beta(\varepsilon), \text { and }\|S-T\|<\varepsilon .
$$

Definition $1.3(9])$. For two Banach spaces $X$ and $Y$, the pair $(X, Y)$ satisfies the Bishop-Phelps-Bollobás property for bilinear forms (for short, BPBP for bilinear forms) if for every $\varepsilon>0$, there are $\eta(\varepsilon)>0$ and $\beta(\varepsilon)>0$ with $\lim _{t \rightarrow 0} \beta(t)=0$ such that for any $A \in S_{L^{2}(X \times Y)}$, if $\left(x_{0}, y_{0}\right) \in S_{X} \times S_{Y}$ is such that $\left|A\left(x_{0}, y_{0}\right)\right|>1-\eta(\varepsilon)$, then there are $B \in S_{L^{2}(X \times Y)}$ and $\left(u_{0}, v_{0}\right) \in S_{X} \times S_{Y}$ satisfying the following conditions:

$$
\left|B\left(u_{0}, v_{0}\right)\right|=1, \quad\left\|u_{0}-x_{0}\right\|<\beta(\varepsilon), \quad\left\|v_{0}-y_{0}\right\|<\beta(\varepsilon) \text { and }\|B-A\|<\varepsilon .
$$

In this definition we can replace $\left(x_{0}, y_{0}\right) \in S_{X} \times S_{Y}$ by $\left(x_{0}, y_{0}\right) \in B_{X} \times B_{Y}$. Also it is not difficult to check that $\lim _{\varepsilon \rightarrow 0^{+}} \eta(\varepsilon)=0$.

We will also consider the BPBP for $n$-linear mappings, which is defined in an analogous way.

The outline of the paper is as follows. Section 2 is devoted to positive results of spaces satisfying the BPBP for $n$-linear or bilinear forms. Indeed if $X_{i}(1 \leq i \leq n)$ 
are uniformly convex Banach spaces, then for every Banach space $Y$, the BPBP for $n$-linear mappings from $X_{1} \times \cdots \times X_{n}$ to $Y$ is satisfied. Up to now, this is the only sufficient condition known that implies BPBP even for bilinear forms. As a consequence of the previous result, one obtains the corresponding condition for operators whose domain is uniformly convex. We already mentioned that the BPBP for bilinear forms on $X \times Y$ implies the BPBP for operators from $X$ into $Y^{*}$, and the converse is no longer true. However, if $Y$ is uniformly convex, then the converse also holds, a result that has also been proved independently by Dai [10. As a consequence, if $Y$ is a uniformly convex Banach space whose dual satisfies some isometric property (called AHSP), then the pair $\left(\ell_{1}, Y\right)$ satisfies the BPBP for bilinear forms. This result can be applied for instance to any $L_{p}(\mu)$ for $1<p<\infty$.

In Section 3 we obtain a characterization of the Banach spaces $Y$ satisfying that the pair $\left(\ell_{1}, Y\right)$ has the BPBP for bilinear forms. In order to do this, we introduce a geometrical property and prove that many classical Banach spaces enjoy this property, including the finite-dimensional normed spaces, uniformly smooth spaces, $\mathcal{C}(K)$ and $K(H)$ (Section 4$)$. On the other hand, the pair $\left(\ell_{1}, L_{1}(\mu)\right)$ does not satisfy the BPBP for bilinear forms for any infinite-dimensional $L_{1}(\mu)$, a result that was known only when $L_{1}(\mu)=\ell_{1}$. Let us notice that the set of norm attaining bilinear forms is dense in $L^{2}\left(\ell_{1} \times L_{1}(\mu)\right)$ (see [15]). For operators, the BPBP in the case $\left(\ell_{1}, L_{\infty}(\mu)\right)$ for every measure $\mu$ is also satisfied (see [1]).

\section{The Bishop-Phelps-Bollobás Theorem for Multilinear mappings ON UNIFORMLY CONVEX BANACH SPACES}

We recall that a Banach space $X$ is uniformly convex if for every $\varepsilon>0$ there is $0<\delta<1$ such that

$$
u, v \in B_{X}, \frac{\|u+v\|}{2}>1-\delta \Rightarrow\|u-v\|<\varepsilon .
$$

In such a case, the modulus of convexity of $X$ is given by

$$
\delta(\varepsilon):=\inf \left\{1-\frac{\|u+v\|}{2}: u, v \in B_{X},\|u-v\| \geq \varepsilon\right\} .
$$

Given a bounded subset $A$ of $X$, an element $x^{*} \in X^{*}$ and $\alpha>0$, the slice $S\left(A, x^{*}, \alpha\right)$ is the subset of $A$ given by

$$
S\left(A, x^{*}, \alpha\right):=\left\{z \in A: \operatorname{Re} x^{*}(z)>\sup _{x \in A} \operatorname{Re} x^{*}(x)-\alpha\right\} .
$$

The following simple lemma will be useful in the proof of the main result in this section.

Lemma 2.1. If $X$ is uniformly convex, then for every $\varepsilon>0$,

$$
\operatorname{diam} S\left(B_{X}, x^{*}, \delta(\varepsilon)\right) \leq \varepsilon, \quad \text { for all } x^{*} \in S_{X^{*}} .
$$

Proof. Indeed if $x^{*} \in S_{X^{*}}$ and we choose $x, z \in S\left(B_{X}, x^{*}, \delta(\varepsilon)\right)$, then

$$
\|x+z\| \geq\left|x^{*}(x+z)\right|>2(1-\delta(\varepsilon)) .
$$

So we deduce $\|x-z\|<\varepsilon$. Since this holds for every pair of elements $x, z$ in the slice, then diam $S\left(B_{X}, x^{*}, \delta(\varepsilon)\right) \leq \varepsilon$, as we wanted to show.

Our aim is now to show that the Bishop-Phelps-Bollobás Theorem holds for $n$ linear mappings defined on uniformly convex Banach spaces. In the next assertion, 
given Banach spaces $X_{j}(1 \leq j \leq n)$ we will denote by $X=\left(X_{1} \times \cdots \times X_{n},\|\cdot\|_{\infty}\right)$, $\|\cdot\|_{\infty}$ being the supremum norm, and by $S$ the set given by

$$
S:=\left\{x=\left(x_{j}\right) \in \prod_{j=1}^{n} X_{j}: x_{j} \in S_{X_{j}}, \text { for all } 1 \leq j \leq n\right\} .
$$

Theorem 2.2. Let $X_{1}, \ldots, X_{n}, Y$ be Banach spaces and assume that every $X_{j}$ is uniformly convex with modulus of convexity $\delta_{j}, 1 \leq j \leq n$. Given $\varepsilon>0$, if $0<\eta<\min \left\{\delta_{j}(\varepsilon): 1 \leq j \leq n\right\} \frac{\varepsilon}{8+2 \varepsilon}$, then for every $A \in S_{L^{n}\left(X_{1} \times \cdots \times X_{n}, Y\right)}$ and every $x_{0} \in S$ such that $\left\|A\left(x_{0}\right)\right\|>1-\eta$, there exist a point $z_{0} \in S$ and $B \in S_{L^{n}\left(X_{1} \times \cdots \times X_{n}, Y\right)}$ satisfying the following conditions:

$$
\left\|B\left(z_{0}\right)\right\|=1, \quad\left\|z_{0}-x_{0}\right\|_{\infty} \leq \varepsilon \text { and }\|B-A\|<\varepsilon .
$$

Moreover, if $A$ belongs to some linear subspace of $L^{n}\left(X_{1} \times \cdots \times X_{n}, Y\right)$ containing the finite-type n-linear mappings, then $B$ belongs to the same subspace.

Proof. Let $0<\varepsilon<1$. Since every $X_{j}$ is uniformly convex, by Lemma 2.1.

$$
\operatorname{diam} S\left(B_{X_{j}}, f_{j}, \delta_{j}(\varepsilon)\right) \leq \varepsilon,
$$

for all $f_{j} \in S_{X_{j}^{*}}$ and every $1 \leq j \leq n$. We define $\alpha:=\min \left\{\delta_{j}(\varepsilon): 1 \leq j \leq n\right\}$ and choose a real number $\eta$ such that

$$
0<\eta<\frac{\alpha \varepsilon}{8+2 \varepsilon}
$$

hence

$$
1+\frac{\varepsilon}{4}\left(1-\frac{\alpha}{2}\right)<\left(1+\frac{\varepsilon}{4}\right)(1-\eta) .
$$

Let $A \in S_{L^{n}\left(X_{1} \times \cdots \times X_{n}, Y\right)}$ and $x_{0}=\left(x_{01}, \ldots, x_{0 n}\right) \in S$ such that $\left\|A\left(x_{0}\right)\right\|>1-\eta$. For each $1 \leq j \leq n$ there is a functional $x_{j}^{*} \in S_{X_{j}^{*}}$ such that $x_{j}^{*}\left(x_{0 j}\right)=1$, and we know that

$$
\operatorname{diam} S\left(B_{X_{j}}, x_{j}^{*}, \alpha\right) \leq \varepsilon, \text { for all } 1 \leq j \leq n .
$$

We define the mapping $C \in L^{n}\left(X_{1} \times \cdots \times X_{n}, Y\right)$ by

$$
C(x):=A(x)+\frac{\varepsilon}{4}\left(\prod_{j=1}^{n} x_{j}^{*}\left(x_{j}\right)\right) A\left(x_{0}\right) \quad\left(x=\left(x_{j}\right) \in X\right) .
$$

Clearly,

and thus

$$
C\left(x_{0}\right)=\left(1+\frac{\varepsilon}{4}\right) A\left(x_{0}\right)
$$

$$
\left\|C\left(x_{0}\right)\right\|=\left(1+\frac{\varepsilon}{4}\right)\left\|A\left(x_{0}\right)\right\|>\left(1+\frac{\varepsilon}{4}\right)(1-\eta) .
$$

Let $\mathbb{T}$ be the set given by $\mathbb{T}:=\{\lambda \in \mathbb{K}:|\lambda|=1\}$. Thus, if $1 \leq j \leq n$ and $z_{j} \in B_{X_{j}} \backslash \mathbb{T} S\left(B_{X_{j}}, x_{j}^{*}, \frac{\alpha}{2}\right)$, it is satisfied that

$$
\left|x_{j}^{*}\left(z_{j}\right)\right| \leq 1-\frac{\alpha}{2}
$$

Hence, if $z \in B_{X}$ and there exists $1 \leq j \leq n$ with $z_{j} \notin \mathbb{T} S\left(B_{X_{j}}, x_{j}^{*}, \frac{\alpha}{2}\right)$, we have that

$$
\|C(z)\| \leq 1+\frac{\varepsilon}{4}\left|x_{j}^{*}\left(z_{j}\right)\right| \leq 1+\frac{\varepsilon}{4}\left(1-\frac{\alpha}{2}\right) .
$$


From (2.2) and the previous inequality it follows that

$$
\left\|C\left(x_{0}\right)\right\|>\|C(z)\|, \text { for all } z \in B_{X} \backslash \prod_{j=1}^{n} \mathbb{T} S\left(B_{X_{j}}, x_{j}^{*}, \frac{\alpha}{2}\right) .
$$

This implies that $\|C\|=\sup \left\{\|C(z)\|: z \in \prod_{j=1}^{n} S\left(B_{X_{j}}, x_{j}^{*}, \frac{\alpha}{2}\right)\right\}$, and

$$
z \in B_{X}, \quad\|C(z)\|>\left(1+\frac{\varepsilon}{4}\right)(1-\eta) \Rightarrow z \in \prod_{j=1}^{n} \mathbb{T} S\left(B_{X_{j}}, x_{j}^{*}, \frac{\alpha}{2}\right) .
$$

It is also clear that

$$
\|C-A\| \leq \frac{\varepsilon}{4} .
$$

By (2.2) we can choose $0<\gamma<\frac{\varepsilon}{4 n}$ such that

$$
\left\|C\left(x_{0}\right)\right\|-\left(1+\frac{\varepsilon}{4}\right)(1-\eta)>n \gamma .
$$

Let us consider the mapping $\phi: B_{X} \longrightarrow \mathbb{R}$ given by

$$
\phi(x):=\|C(x)\| \quad\left(x \in B_{X}\right),
$$

which is continuous and bounded. Since $X_{j}$ is uniformly convex for every $1 \leq j \leq n$, the space $X$ is reflexive, so it has the Radon-Nikodým property. Hence $B_{X}$ is a Radon-Nikodým set and we can apply [19, Theorem 14] to obtain an element $u_{0} \in B_{X}$ and functionals $z_{j}^{*} \in X_{j}^{*}(1 \leq j \leq n)$ such that

$$
0<\left\|z_{j}^{*}\right\| \leq \gamma, \text { for all } 1 \leq j \leq n
$$

and the function

$$
x=\left(x_{1}, \ldots, x_{n}\right) \mapsto\|C(x)\|+\sum_{j=1}^{n} \operatorname{Re} z_{j}^{*}\left(x_{j}\right) \quad\left(x=\left(x_{j}\right) \in B_{X}\right)
$$

attains its maximum at $u_{0} \in B_{X}$. By using that $C$ is an $n$-linear mapping and the unit ball of a Banach space is balanced, it is immediate to deduce that

$$
u_{0 j} \in S_{X_{j}}, \quad \operatorname{Re} z_{j}^{*}\left(u_{0 j}\right)=z_{j}^{*}\left(u_{0 j}\right)=\left|z_{j}^{*}\left(u_{0 j}\right)\right|, \text { for all } 1 \leq j \leq n,
$$

and indeed it is satisfied that

$\|C(x)\| \leq\|C(x)\|+\sum_{j=1}^{n}\left|z_{j}^{*}\left(x_{j}\right)\right| \leq\left\|C\left(u_{0}\right)\right\|+\sum_{j=1}^{n}\left|z_{j}^{*}\left(u_{0 j}\right)\right|$, for all $x=\left(x_{j}\right) \in B_{X}$.

As a consequence $\|C\| \leq\left\|C\left(u_{0}\right)\right\|+\sum_{j=1}^{n}\left|z_{j}^{*}\left(u_{0 j}\right)\right|$, and so by using (2.6) and (2.5) we have that

$$
\left\|C\left(u_{0}\right)\right\| \geq\|C\|-n \gamma>\left(1+\frac{\varepsilon}{4}\right)(1-\eta)>0 .
$$

By now using (2.3) we obtain

$$
u_{0} \in \prod_{j=1}^{n} \mathbb{T} S\left(B_{X_{j}}, x_{j}^{*}, \frac{\alpha}{2}\right)
$$


Let us write $y_{0}:=\frac{C\left(u_{0}\right)}{\left\|C\left(u_{0}\right)\right\|}$, and for each $1 \leq j \leq n$ choose $u_{j}^{*} \in S_{X_{j}^{*}}$ such that $u_{j}^{*}\left(u_{0 j}\right)=1$. Now we define

$$
D(x):=C(x)+\left(\sum_{j=1}^{n} z_{j}^{*}\left(x_{j}\right) \prod_{\substack{i=1 \\ i \neq j}}^{n} u_{i}^{*}\left(x_{i}\right)\right) y_{0} \quad\left(x=\left(x_{j}\right) \in B_{X}\right) .
$$

It is clear that $D \in L^{n}\left(X_{1} \times \cdots \times X_{n}, Y\right)$ and

$$
\|D-C\| \leq \sum_{j=1}^{n}\left\|z_{j}^{*}\right\| \leq n \gamma<\frac{\varepsilon}{4}
$$

For any $x=\left(x_{j}\right) \in B_{X}$ we have that

$$
\begin{aligned}
\|D(x)\| & \leq\|C(x)\|+\sum_{j=1}^{n}\left|z_{j}^{*}\left(x_{j}\right)\right|\left(\prod_{\substack{i=1 \\
i \neq j}}^{n}\left\|u_{i}^{*}\right\|\right) \\
& \leq\|C(x)\|+\sum_{j=1}^{n}\left|z_{j}^{*}\left(x_{j}\right)\right| \\
& \leq\left\|C\left(u_{0}\right)\right\|+\sum_{j=1}^{n}\left|z_{j}^{*}\left(u_{0 j}\right)\right| .
\end{aligned}
$$

Also it is satisfied that

$$
\begin{aligned}
\left\|D\left(u_{0}\right)\right\| & =\left\|C\left(u_{0}\right)+\sum_{j=1}^{n} z_{j}^{*}\left(u_{0 j}\right) y_{0}\right\| \\
& =\left\|C\left(u_{0}\right)+\sum_{j=1}^{n}\left|z_{j}^{*}\left(u_{0 j}\right)\right| y_{0}\right\| \\
& =\left\|C\left(u_{0}\right)\right\|+\sum_{j=1}^{n}\left|z_{j}^{*}\left(u_{0 j}\right)\right| .
\end{aligned}
$$

As a consequence $D$ attains its norm at $u_{0}$.

In view of (2.7), for every $1 \leq j \leq n$ there is $\lambda_{j} \in \mathbb{T}$ such that $\lambda_{j} u_{0 j} \in$ $S\left(B_{X_{j}}, x_{j}^{*}, \frac{\alpha}{2}\right)$. By using (2.1) we deduce that

$$
\left\|\lambda_{j} u_{0 j}-x_{0 j}\right\| \leq \operatorname{diam} S\left(B_{X_{j}}, x_{j}^{*}, \frac{\alpha}{2}\right) \leq \varepsilon, \text { for all } 1 \leq j \leq n .
$$

If we write $z_{0}:=\left(\lambda_{j} u_{0 j}\right)$, then $z_{0} \in B_{X}, D$ attains its norm at $z_{0}$ and

$$
\left\|z_{0}-x_{0}\right\|_{\infty} \leq \varepsilon \text {. }
$$

Let us notice that $D-A$ is an $n$-linear mapping of finite type. Indeed it is the sum of (at most) $n+1 n$-linear mappings of the form

$$
x \mapsto \prod_{j=1}^{n} x_{j}^{*}\left(x_{j}\right) y \quad\left(x=\left(x_{j}\right) \in X\right),
$$

where $x_{j}^{*} \in X_{j}^{*}$ for every $j$ and $y \in Y$. 
By taking $B:=\frac{D}{\|D\|}$ we have that $B \in S_{L^{n}\left(X_{1} \times \cdots \times X_{n}, Y\right)}, B$ attains its norm at $z_{0}$ and

$$
\begin{gathered}
\|B-A\| \leq\|B-D\|+\|D-A\| \\
\leq|1-\|D\||+\|D-A\| \leq 2\|D-A\| \\
\leq 2(\|D-C\|+\|C-A\|)<\varepsilon \quad(\text { by (2.8) and (2.4) }) .
\end{gathered}
$$

We have proved that the BPBP for $n$-linear mappings from $X_{1} \times \cdots \times X_{n}$ to $Y$ is satisfied.

We deduce the following immediate consequence for operators:

Corollary 2.3. Let $X$ and $Y$ be Banach spaces, and assume that $X$ is uniformly convex. Then given $\varepsilon>0$, there is $\eta>0$ such that for every $R \in S_{L(X, Y)}$ and $x_{0} \in S_{X}$ such that $\left\|R\left(x_{0}\right)\right\|>1-\eta$, there exist a point $u_{0} \in S_{X}$ and $T \in S_{L(X, Y)}$ satisfying the following conditions:

$$
\left\|T\left(u_{0}\right)\right\|=1, \quad\left\|u_{0}-x_{0}\right\| \leq \varepsilon \text { and }\|T-R\|<\varepsilon .
$$

Actually any $0<\eta<\frac{\varepsilon \delta(\varepsilon)}{8+2 \varepsilon}$ satisfies the above condition.

In addition, if $R$ belongs to some linear space $M \subset L(X, Y)$ containing the finite-rank operators, then $T$ also belongs to $M$.

As we already mentioned, the BPBP for operators from $X$ into $Y^{*}$ is satisfied if the pair $(X, Y)$ satisfies the BPBP for bilinear forms and the converse is not true. However, we will provide a class of Banach spaces for which the converse holds. The next result has also been proved independently by Dai [10, Theorem 3.1].

Proposition 2.4. Let $X$ and $Y$ be Banach spaces and assume that $Y$ is uniformly convex. If the pair $\left(X, Y^{*}\right)$ has the Bishop-Phelps-Bollobás property for operators, then $(X, Y)$ satisfies the Bishop-Phelps-Bollobás property for bilinear forms.

Proof. Given $\varepsilon_{0}>0$, we can see that the pair $\left(X, Y^{*}\right)$ has the BPBP for operators for $\varepsilon>0$ small enough such that $\max \{\varepsilon, \beta(\varepsilon)\}<\min \left\{\varepsilon_{0}, \frac{1}{3} \delta\left(\varepsilon_{0}\right)\right\}$, where $\delta\left(\varepsilon_{0}\right)$ is the modulus of convexity of $Y$. Let $0<\eta<\min \left\{\eta(\varepsilon), \frac{1}{3} \delta\left(\varepsilon_{0}\right)\right\}$. Take $A \in S_{L^{2}(X \times Y)}$ and assume that $\left(x_{0}, y_{0}\right) \in S_{X} \times S_{Y}$ is such that $\left|A\left(x_{0}, y_{0}\right)\right|>1-\eta$. By rotating $A$ if necessary, we can assume that $\left|A\left(x_{0}, y_{0}\right)\right|=A\left(x_{0}, y_{0}\right)$. If $T \in L\left(X, Y^{*}\right)$ is the operator associated to $A$, then we know that

$$
\|T\|=\|A\|=1, \text { and } \operatorname{Re} T\left(x_{0}\right)\left(y_{0}\right)=\operatorname{Re} A\left(x_{0}, y_{0}\right)>1-\eta .
$$

By assumption, there are an operator $S \in S_{L\left(X, Y^{*}\right)}$ and an element $z_{0} \in S_{X}$ satisfying

$$
\left\|S\left(z_{0}\right)\right\|=1, \quad\left\|z_{0}-x_{0}\right\|<\beta(\varepsilon), \quad\|S-T\|<\varepsilon .
$$

As a consequence we have

$$
\begin{gathered}
\operatorname{Re} S\left(z_{0}\right)\left(y_{0}\right)>\operatorname{Re} T\left(z_{0}\right)\left(y_{0}\right)-\varepsilon>\operatorname{Re} T\left(x_{0}\right)\left(y_{0}\right)-\varepsilon-\left\|z_{0}-x_{0}\right\| \\
>1-\eta-\varepsilon-\beta(\varepsilon)>1-\delta\left(\varepsilon_{0}\right) .
\end{gathered}
$$

Since $Y$ is uniformly convex, then $Y$ is reflexive, and so there is $u_{0} \in S_{Y}$ such that $S\left(z_{0}\right)\left(u_{0}\right)=1$.

Therefore we have

$$
1-\frac{\delta\left(\varepsilon_{0}\right)}{2}<\operatorname{Re} \frac{S\left(z_{0}\right)\left(u_{0}+y_{0}\right)}{2} \leq\left\|S z_{0}\right\| \frac{\left\|u_{0}+y_{0}\right\|}{2} \leq \frac{\left\|u_{0}+y_{0}\right\|}{2} .
$$


By using that $Y$ is uniformly convex we deduce

$$
\left\|u_{0}-y_{0}\right\|<\varepsilon_{0} .
$$

Hence, if we denote by $B$ the bilinear form associated to $S$, then we have that

$$
\begin{gathered}
B\left(z_{0}, u_{0}\right)=S\left(z_{0}\right)\left(u_{0}\right)=1=\|B\|, \\
\|B-A\|<\varepsilon_{0}, \quad\left\|z_{0}-x_{0}\right\|<\beta(\varepsilon)<\varepsilon_{0}, \quad\left\|u_{0}-y_{0}\right\|<\varepsilon_{0} .
\end{gathered}
$$

So the bilinear form $B$ satisfies all the required conditions.

Now we will recall the isometric property that was already used in [1] to describe the Banach spaces $Y$ such that the pair $\left(\ell_{1}, Y\right)$ satisfies the BPBP for operators.

Definition 2.5 ([1, Remark 3.2]). A Banach space $X$ is said to have the Approximate Hyperplane Series property (for short, AHSP) if for every $\varepsilon>0$ there exist $0<\eta, \delta<\varepsilon$ such that for every sequence $\left(x_{k}\right) \subset S_{X}$ and every convex series $\sum_{k=1}^{\infty} \alpha_{k}$ with

$$
\left\|\sum_{k=1}^{\infty} \alpha_{k} x_{k}\right\|>1-\eta,
$$

there exist a subset $A \subset \mathbb{N}$, a subset $\left\{z_{k}: k \in A\right\} \subset S_{X}$ and $x^{*} \in S_{X^{*}}$ satisfying

(1) $\sum_{k \in A} \alpha_{k}>1-\delta$, and

(2) (a) $\left\|z_{k}-x_{k}\right\|<\varepsilon$ for all $k \in A$,

(b) $x^{*}\left(z_{k}\right)=1$ for each $k \in A$.

Corollary 2.6. If $Y$ is a uniformly convex Banach space and $Y^{*}$ has the Approximate Hyperplane Series property, then the pair $\left(\ell_{1}, Y\right)$ has the Bishop-PhelpsBollobás property for bilinear forms.

The previous statement is a consequence of the fact that the pair $\left(\ell_{1}, Y^{*}\right)$ has the BPBP for operators only when $Y^{*}$ has the AHSP (see [1, Theorem 4.1]) and Proposition 2.4. Examples of classes of spaces having the AHSP can be found in [1] and [7. For instance, it is known that the finite-dimensional spaces, $\mathcal{C}(K)$, $L_{1}(\mu)$ and uniform convex Banach spaces have this property. Indeed, every almost CL-space satisfies the AHSP (see for instance [13] for the definitions and also [7]). Furthermore every lush space has the AHSP (see [8]). Also, spaces whose dual norm satisfies some uniform condition of smoothness (USSD) at some boundary have this property (see [12] and [7]).

\section{The Bishop-Phelps-Bollobás Theorem for Bilinear Mappings ON THE PRODUCT OF $\ell_{1}$ AND ANOTHER BANACH SPACE}

By looking directly at the proof of the BPBP for bilinear forms on the product $\ell_{1} \times Y$ ( $Y$ a Banach space $)$ we will obtain a more general result than the one appearing in Corollary 2.6. The Banach spaces $Y$ such that the pair $\left(\ell_{1}, Y\right)$ satisfies the BPBP for bilinear forms do have a geometric property that we will characterize in this section.

Definition 3.1. For a Banach space $Y$ we will say that the pair $\left(Y, Y^{*}\right)$ satisfies the Approximate Hyperplane Series property (for short, AHSP) if for every $\varepsilon>0$ there are $0<\delta, \eta<\varepsilon$ satisfying that for every convex series $\sum_{n} \alpha_{n}$ and for every sequence 
of functionals $\left\{y_{n}^{*}\right\}$ in $S_{Y^{*}}$ and $y_{0} \in S_{Y}$ such that Re $\sum_{n} \alpha_{n} y_{n}^{*}\left(y_{0}\right)>1-\eta$, there are a subset $C \subset \mathbb{N},\left\{z_{k}^{*}: k \in C\right\} \subset S_{Y^{*}}$ and $z_{0} \in S_{Y}$ such that

$$
\sum_{k \in C} \alpha_{k}>1-\delta,\left\|z_{k}^{*}-y_{k}^{*}\right\|<\varepsilon, \quad z_{k}^{*}\left(z_{0}\right)=1, \text { for all } k \in C \text { and }\left\|z_{0}-y_{0}\right\|<\varepsilon \text {. }
$$

It is not difficult to check that by assuming in Definition 3.1 that the sequence $\left\{y_{n}^{*}\right\}$ is contained in $B_{Y^{*}}$, an equivalent condition is obtained. It is also clear that if the pair $\left(Y, Y^{*}\right)$ has the AHSP, then $Y^{*}$ has the AHSP. As we will see later, as a consequence of our results and previous work, both properties are not equivalent (see Proposition 4.8).

The following elementary result is slightly more general than [1, Lemma 3.3], and the proof is almost the same.

Lemma 3.2. Let $\left\{c_{n}\right\}$ be a sequence of complex numbers with $\left|c_{n}\right| \leq 1$ for every $n$, let $\eta>0$ and let $\left\{\alpha_{n}\right\}$ be a sequence of nonnegative real numbers such that $\sum_{n=1}^{\infty} \alpha_{n} \leq 1$. Assume also that $\operatorname{Re} \sum_{n=1}^{\infty} \alpha_{n} c_{n}>1-\eta$. Then for every $0<r<1$, the set $A:=\left\{i \in \mathbb{N}: \operatorname{Re} c_{i}>r\right\}$ satisfies the estimate

$$
\sum_{i \in A} \alpha_{i}>1-\frac{\eta}{1-r} .
$$

The following result will be useful in order to provide examples of spaces $Y$ satisfying that the pair $\left(Y, Y^{*}\right)$ has the AHSP. It has the advantage that the use of convex series is avoided.

Proposition 3.3. Let $Y$ be a Banach space. If for every $\varepsilon>0$ there is $\delta>0$ satisfying that for every finite set $F$ and for every finite sequence of functionals $\left\{y_{i}^{*}: i \in F\right\} \subset S_{Y^{*}}$ and $y_{0} \in S_{Y}$ such that Re $y_{i}^{*}\left(y_{0}\right)>1-\delta$, for every $i \in F$ there are $\left\{z_{k}^{*}: i \in F\right\} \subset S_{Y^{*}}$ and $z_{0} \in S_{Y}$ such that

$$
\left\|z_{i}^{*}-y_{i}^{*}\right\|<\varepsilon, \quad z_{i}^{*}\left(z_{0}\right)=1, \quad \text { for all } i \in F, \quad \text { and }\left\|z_{0}-y_{0}\right\|<\varepsilon,
$$

then the pair $\left(Y, Y^{*}\right)$ satisfies the Approximate Hyperplane Series property.

Proof. Given $\varepsilon>0$ there is $\delta>0$ satisfying the assumption and we can clearly assume $\delta<\varepsilon<1$. Let us consider a convex series $\sum \alpha_{n}$, a sequence $\left\{y_{n}^{*}\right\}$ in $S_{Y^{*}}$ and $y_{0} \in S_{Y}$ such that

$$
\operatorname{Re} \sum_{n=1}^{\infty} \alpha_{n} y_{n}^{*}\left(y_{0}\right)>1-\delta^{2},
$$

and choose $N$ large enough such that Re $\sum_{k=1}^{N} \alpha_{k} y_{k}^{*}\left(y_{0}\right)>1-\delta^{2}$. Let us define

$$
C:=\left\{n \in \mathbb{N}: n \leq N, \operatorname{Re} y_{n}^{*}\left(y_{0}\right)>1-\delta\right\} .
$$

By Lemma 3.2 applied with $\eta=\delta^{2}$ and $r=1-\delta$, we obtain

$$
\sum_{n \in C} \alpha_{n}>1-\delta
$$

By using the assumption for the finite set $C$ we obtain a finite set

$$
\left\{z_{i}^{*}: i \in C\right\} \subset S_{Y^{*}}, \text { and an element } z_{0} \in S_{Y},
$$

satisfying

$$
\left\|z_{i}^{*}-y_{i}^{*}\right\|<\varepsilon, \quad z_{i}^{*}\left(z_{0}\right)=1, \text { for all } i \in C,\left\|z_{0}-y_{0}\right\|<\varepsilon,
$$

and we also know that $\sum_{n \in C} \alpha_{n}>1-\delta$, as we wanted to show. 
Let us notice that the above condition is a version of the Bishop-Phelps-Bollobás Theorem which uses any finite set of functionals instead of one. We will see later that this change really makes a difference.

For a Banach space $X$ and $x \in S_{X}$, we will denote

$$
D(x):=\left\{x^{*} \in S_{X^{*}}: x^{*}(x)=1\right\} .
$$

The set-valued mapping $D: S_{X} \mapsto S_{X^{*}}$ is called the duality mapping of $X$. It is clear that from Proposition 3.3 the following result can be deduced.

Corollary 3.4. Let $X$ be a Banach space. If for every $\varepsilon>0$ there is $\delta>0$ such that for every $x \in S_{X}$ there is $y \in S_{X}$ satisfying

(1) $\|y-x\|<\varepsilon$,

(2) if $x^{*} \in S_{X^{*}}$ satisfies $\operatorname{Re} x^{*}(x)>1-\delta$, then $\operatorname{dist}\left(x^{*}, D(y)\right)<\varepsilon$, then the pair $\left(X, X^{*}\right)$ satisfies the Approximate Hyperplane Series property.

Now, we characterize the Banach spaces $Y$ satisfying that the pair $\left(\ell_{1}, Y\right)$ has the BPBP for bilinear forms. The following elementary fact will be useful for this purpose.

Lemma 3.5. Let $z$ be a complex number with $|z| \leq 1$ and $0<r<1$. If $\operatorname{Re} z>r$, then $|z-1|^{2}<2(1-r)$.

Proof. It is clear that

$$
\begin{gathered}
|z-1|^{2}=(\operatorname{Re} z-1)^{2}+\operatorname{Im}^{2} z \\
=\operatorname{Re}^{2} z+\operatorname{Im}^{2} z+1-2 \operatorname{Re} z \\
\quad \leq 2(1-\operatorname{Re} z)<2(1-r) .
\end{gathered}
$$

Theorem 3.6. Let $Y$ be a Banach space. Then the pair $\left(\ell_{1}, Y\right)$ has the BishopPhelps-Bollobás property for bilinear forms if and only if the pair $\left(Y, Y^{*}\right)$ satisfies the Approximate Hyperplane Series property.

Proof. Assume that the pair $\left(\ell_{1}, Y\right)$ has the BPBP for bilinear forms. Then for every $\varepsilon>0$ there are $\beta(\varepsilon)$ and $\eta(\varepsilon)$ satisfying the conditions of Definition 1.3 .

Given $0<\varepsilon_{0}<1$, we are going to show that $\left(Y, Y^{*}\right)$ has the AHSP for $\delta=$ $\beta(\varepsilon)+\sqrt{\beta(\varepsilon)}$ and $\eta=\eta(\varepsilon)$, where $\varepsilon>0$ is so that

$$
3 \sqrt{2(\eta(\varepsilon)+2 \beta(\varepsilon)+\varepsilon)}+(4 \beta(\varepsilon))^{\frac{1}{4}}+\varepsilon<\varepsilon_{0} .
$$

Let us take a convex series $\sum_{n} \alpha_{n}$, a sequence of functionals $\left\{y_{n}^{*}\right\}$ in $S_{Y^{*}}$ and $y_{0} \in S_{Y}$ such that $\operatorname{Re} \sum_{n} \alpha_{n} y_{n}^{*}\left(y_{0}\right)>1-\eta(\varepsilon)$.

Now we define the bilinear form $A$ on $\ell_{1} \times Y$ given by

$$
A(x, y)=\sum_{n=1}^{\infty} x(n) y_{n}^{*}(y) \quad\left((x, y) \in \ell_{1} \times Y, x=(x(n))\right) .
$$

It is clear that $A$ is well defined and, moreover, $A \in S_{L^{2}\left(\ell_{1} \times Y\right)}$ since for every $(x, y) \in \ell_{1} \times Y$ it holds that

$$
|A(x, y)| \leq \sum_{n=1}^{\infty}|x(n)|\left|y_{n}^{*}(y)\right| \leq \sum_{n=1}^{\infty}|x(n)|\|y\|=\|x\|_{1}\|y\|
$$


and also $A\left(e_{1}, y\right)=y_{1}^{*}(y)$, so $\|A\| \geq \sup _{y \in S_{Y}}\left|y_{1}^{*}(y)\right|=\left\|y_{1}^{*}\right\|=1$. Here $\|\cdot\|_{1}$ denotes the usual norm and $\left(e_{n}\right)$ the canonical basis in $\ell_{1}$.

The condition $\operatorname{Re} \sum_{n} \alpha_{n} y_{n}^{*}\left(y_{0}\right)>1-\eta(\varepsilon)$ implies that the element $x_{0}=\left(\alpha_{n}\right)$ satisfies $\left|A\left(x_{0}, y_{0}\right)\right|>1-\eta(\varepsilon)$. By assumption we can find elements $\left(u_{0}, v_{0}\right) \in$ $S_{\ell_{1}} \times S_{Y}$ and a bilinear form $B \in S_{L^{2}\left(\ell_{1} \times Y\right)}$ such that

$$
\|B-A\|<\varepsilon, \quad\left\|u_{0}-x_{0}\right\|_{1}<\beta(\varepsilon), \quad\left\|v_{0}-y_{0}\right\|<\beta(\varepsilon), \quad\left|B\left(u_{0}, v_{0}\right)\right|=1 .
$$

Since $\left|B\left(u_{0}, v_{0}\right)\right|=1$, there is a real number $\theta \in \mathbb{R}$ satisfying $e^{i \theta}=B\left(u_{0}, v_{0}\right)$. We clearly have that

$$
\begin{gathered}
1=\left|B\left(u_{0}, v_{0}\right)\right|=e^{-i \theta} B\left(u_{0}, v_{0}\right)=\operatorname{Re} e^{-i \theta} B\left(u_{0}, v_{0}\right) \\
=\operatorname{Re}\left(\sum_{u_{0}(n) \neq 0}\left|u_{0}(n)\right| e^{-i \theta} \frac{u_{0}(n)}{\left|u_{0}(n)\right|} B\left(e_{n}, v_{0}\right)\right) \\
=\sum_{u_{0}(n) \neq 0}\left|u_{0}(n)\right| \operatorname{Re}\left(e^{-i \theta} \frac{u_{0}(n)}{\left|u_{0}(n)\right|} B\left(e_{n}, v_{0}\right)\right) \\
\leq \sum_{u_{0}(n) \neq 0}\left|u_{0}(n)\right|=1 .
\end{gathered}
$$

It follows that

$$
\begin{aligned}
n \in \mathbb{N}, u_{0}(n) \neq 0 & \Rightarrow \operatorname{Re}\left(e^{-i \theta} \frac{u_{0}(n)}{\left|u_{0}(n)\right|} B\left(e_{n}, v_{0}\right)\right)=1 \\
& \Rightarrow \frac{u_{0}(n)}{\left|u_{0}(n)\right|} B\left(e_{n}, v_{0}\right)=e^{i \theta} .
\end{aligned}
$$

By using (3.3) we also have that

$$
\begin{gathered}
1-\beta(\varepsilon)=e^{-i \theta} B\left(u_{0}, v_{0}\right)-\beta(\varepsilon) \\
<\operatorname{Re} e^{-i \theta} B\left(x_{0}, v_{0}\right)=\operatorname{Re} \sum_{n=1}^{\infty} \alpha_{n} e^{-i \theta} B\left(e_{n}, v_{0}\right) .
\end{gathered}
$$

Since we fixed $\varepsilon$ at the beginning, we write $r:=1-\sqrt{\beta(\varepsilon)}$ and define

$$
H:=\left\{n \in \mathbb{N}: \operatorname{Re} e^{-i \theta} B\left(e_{n}, v_{0}\right)>r\right\} .
$$

In view of Lemma 3.2 we obtain that

$$
\sum_{n \in H} \alpha_{n}>1-\sqrt{\beta(\varepsilon)}
$$

Now we define $C:=H \cap\left\{n \in \mathbb{N}: u_{0}(n) \neq 0\right\}$. Then we deduce that

$$
\begin{gathered}
\sum_{n \in C} \alpha_{n}=\sum_{n \in H} \alpha_{n}-\sum_{\substack{n \in H \\
u_{0}(n)=0}} \alpha_{n} \geq \sum_{n \in H} \alpha_{n}-\left\|u_{0}-x_{0}\right\|_{1} \\
>1-\sqrt{\beta(\varepsilon)}-\beta(\varepsilon)=1-\delta .
\end{gathered}
$$

Now it suffices to give the functionals $\left\{z_{n}^{*}: n \in C\right\}$ that will satisfy the condition needed in order to prove that the pair $\left(Y, Y^{*}\right)$ has the AHSP. For this purpose we will consider appropriate small perturbations of the functionals $y \mapsto B\left(e_{n}, y\right)$.

Let us fix $n \in C$. Since $\|B\|=1$ and $\operatorname{Re} e^{-i \theta} B\left(e_{n}, v_{0}\right)>r=1-\sqrt{\beta(\varepsilon)}$, by Lemma 3.5 we deduce that

$$
\left|e^{-i \theta} B\left(e_{n}, v_{0}\right)-1\right|^{2}<2 \sqrt{\beta(\varepsilon)} .
$$


On the other hand, by using (3.3) it follows that

$$
\begin{gathered}
\operatorname{Re} e^{i \theta}=\operatorname{Re} B\left(u_{0}, v_{0}\right)>\operatorname{Re} A\left(u_{0}, v_{0}\right)-\|A-B\| \\
>\operatorname{Re} A\left(x_{0}, v_{0}\right)-\left\|x_{0}-u_{0}\right\|_{1}-\|A-B\| \\
>\operatorname{Re} A\left(x_{0}, y_{0}\right)-\left\|y_{0}-v_{0}\right\|-\left\|x_{0}-u_{0}\right\|_{1}-\|A-B\| \\
>1-\eta(\varepsilon)-2 \beta(\varepsilon)-\varepsilon .
\end{gathered}
$$

Now we can apply Lemma 3.5 to obtain that

$$
\left|e^{i \theta}-1\right|^{2}<2(\eta(\varepsilon)+2 \beta(\varepsilon)+\varepsilon) .
$$

By again applying Lemma 3.5 we also deduce that

$$
n \in H \Rightarrow\left|e^{-i \theta} B\left(e_{n}, v_{0}\right)-1\right|^{2}<2 \sqrt{\beta(\varepsilon)} .
$$

Hence for every $n \in C$, in view of $(\underline{3.4})$ we obtain that

$$
\begin{gathered}
\left|\frac{u_{0}(n)}{\left|u_{0}(n)\right|}-1\right| \leq\left|\frac{u_{0}(n)}{\left|u_{0}(n)\right|}-\frac{u_{0}(n)}{\left|u_{0}(n)\right|} B\left(e_{n}, v_{0}\right)\right|+\left|\frac{u_{0}(n)}{\left|u_{0}(n)\right|} B\left(e_{n}, v_{0}\right)-1\right| \\
=\left|1-B\left(e_{n}, v_{0}\right)\right|+\left|e^{i \theta}-1\right| \\
\leq\left|1-e^{i \theta}\right|+\left|e^{i \theta}-B\left(e_{n}, v_{0}\right)\right|+\left|1-e^{i \theta}\right| \\
\left.<2 \sqrt{2(\eta(\varepsilon)+2 \beta(\varepsilon)+\varepsilon)}+\left|1-e^{-i \theta} B\left(e_{n}, v_{0}\right)\right| \text { (by (3.8) }\right), \\
\left.<2 \sqrt{2(\eta(\varepsilon)+2 \beta(\varepsilon)+\varepsilon)}+(4 \beta(\varepsilon))^{\frac{1}{4}} \text { (by (3.9) }\right) .
\end{gathered}
$$

That is,

$$
\left|\frac{u_{0}(n)}{\left|u_{0}(n)\right|}-1\right| \leq 2 \sqrt{2(\eta(\varepsilon)+2 \beta(\varepsilon)+\varepsilon)}+(4 \beta(\varepsilon))^{\frac{1}{4}}, \quad \text { for all } n \in C .
$$

Finally we define the functionals $z_{n}^{*}$ by

$$
z_{n}^{*}(y):=\frac{u_{0}(n)}{\left|u_{0}(n)\right|} e^{-i \theta} B\left(e_{n}, y\right) \text { and } v_{n}^{*}(y):=B\left(e_{n}, y\right) \quad(n \in C) .
$$

Clearly it is satisfied that $\left\{z_{n}^{*}: n \in C\right\} \subset S_{Y^{*}}$, and in view of (3.4) we know that

$$
z_{n}^{*}\left(v_{0}\right)=1, \quad \text { for all } n \in C .
$$

Also we have that

$$
\begin{gathered}
\left\|z_{n}^{*}-y_{n}^{*}\right\|=\left\|\frac{u_{0}(n)}{\left|u_{0}(n)\right|} e^{-i \theta} v_{n}^{*}-y_{n}^{*}\right\| \\
\leq\left\|\frac{u_{0}(n)}{\left|u_{0}(n)\right|} e^{-i \theta} v_{n}^{*}-e^{-i \theta} v_{n}^{*}\right\|+\left\|e^{-i \theta} v_{n}^{*}-v_{n}^{*}\right\|+\left\|v_{n}^{*}-y_{n}^{*}\right\| \\
\leq\left|\frac{u_{0}(n)}{\left|u_{0}(n)\right|}-1\right|+\left|e^{-i \theta}-1\right|+\|B-A\| \\
\left.\leq 3 \sqrt{2(\eta(\varepsilon)+2 \beta(\varepsilon)+\varepsilon)}+(4 \beta(\varepsilon))^{\frac{1}{4}}+\varepsilon<\varepsilon_{0} \quad \text { (by (3.10) } \text { (3.8) and (3.3) }\right) .
\end{gathered}
$$

In view of (3.6), the above inequality, (3.3) and (3.11) we have proved that the pair $\left(Y, Y^{*}\right)$ has the AHSP.

Conversely, assume that $\left(Y, Y^{*}\right)$ satisfies the AHSP. Given $0<\varepsilon<1$, there are $0<\delta, \eta<\frac{\varepsilon}{2}$ satisfying the conditions in Definition 3.1 for $\frac{\varepsilon}{2}$. Suppose that $A \in S_{L^{2}\left(\ell_{1} \times Y\right)}$ and for some pair $\left(x_{0}, y_{0}\right) \in S_{\ell_{1}} \times S_{Y}$ it holds that $\left|A\left(x_{0}, y_{0}\right)\right|>1-\eta$. By rotating $A$ if necessary we can assume that $A\left(x_{0}, y_{0}\right)>0$. Also, by applying a 
convenient isometry on $\ell_{1}$ we may assume that $x_{0}(n) \geq 0$ for every natural number $n$. Hence we have that

$$
\operatorname{Re} \sum_{n=1}^{\infty} x_{0}(n) A\left(e_{n}, y_{0}\right)>1-\eta .
$$

Since $\|A\|=1$, the sequence of functionals $\left\{y_{n}^{*}\right\}$ given by $y_{n}^{*}(y):=A\left(e_{n}, y\right), n \in \mathbb{N}$, is a subset of $B_{Y^{*}}$. By using the AHSP for the pair $\left(Y, Y^{*}\right)$ we can find a subset $C \subset \mathbb{N}$ such that

$$
\sum_{n \in C} x_{0}(n)>1-\delta
$$

and a subset of functionals $\left\{z_{k}^{*}: k \in C\right\} \subset S_{Y^{*}}$ and $z_{0} \in S_{Y}$ such that

$$
\left\|z_{k}^{*}-y_{k}^{*}\right\|<\frac{\varepsilon}{2}, \quad z_{k}^{*}\left(z_{0}\right)=1, \quad \text { for all } k \in C \quad \text { and } \quad\left\|z_{0}-y_{0}\right\|<\frac{\varepsilon}{2} .
$$

Now we define the bilinear form $B$ on $\ell_{1} \times Y$ given by

$$
B(x, y)=\sum_{n \in C} x(n) z_{n}^{*}(y)+\sum_{n \notin C} x(n) A\left(e_{n}, y\right) \quad\left((x, y) \in \ell_{1} \times Y, x=(x(n))\right) .
$$

It is immediate to check that $B$ is bounded since $\left\|z_{n}^{*}\right\|=1$ for every $n \in C$ and $\|A\|=1$. Since $C$ is nonempty, it is easy to deduce that $B \in S_{L^{2}\left(\ell_{1} \times Y\right)}$. Also,

$$
\begin{gathered}
\|B-A\| \leq \sup _{n} \sup _{y \in B_{Y}}\left|(B-A)\left(e_{n}, y\right)\right| \\
=\sup _{n \in C} \sup _{y \in B_{Y}}\left|(B-A)\left(e_{n}, y\right)\right| \\
=\sup _{n \in C}\left\|z_{n}^{*}-y_{n}^{*}\right\| \leq \frac{\varepsilon}{2}<\varepsilon .
\end{gathered}
$$

Let us take $u_{0}:=\frac{1}{\sum_{n \in C} x_{0}(n)} \sum_{n \in C} x_{0}(n) e_{n}$ that satisfies $u_{0} \in S_{\ell_{1}}$. Also,

$$
\begin{gathered}
\left\|u_{0}-x_{0}\right\|_{1}=\left\|\frac{1}{\sum_{n \in C} x_{0}(n)} \sum_{n \in C} x_{0}(n) e_{n}-x_{0}\right\|_{1} \\
\leq\left\|\frac{1}{\sum_{n \in C} x_{0}(n)} \sum_{n \in C} x_{0}(n) e_{n}-\sum_{n \in C} x_{0}(n) e_{n}\right\|_{1}+\left\|\sum_{n \notin C} x_{0}(n) e_{n}\right\| \\
=1-\sum_{n \in C} x_{0}(n)+\sum_{n \notin C} x_{0}(n)<2 \delta<\varepsilon .
\end{gathered}
$$

Finally we have that

$$
\begin{gathered}
B\left(u_{0}, z_{0}\right)=\sum_{n \in C} u_{0}(n) B\left(e_{n}, z_{0}\right) \\
=\frac{1}{\sum_{n \in C} x_{0}(n)} \sum_{n \in C} x_{0}(n) z_{n}^{*}\left(z_{0}\right)=1 .
\end{gathered}
$$

Therefore $\left(\ell_{1}, Y\right)$ has the BPBP for bilinear forms. 


\section{Banach spaces $X$ such that $\left(X, X^{*}\right)$ has the Approximate HyPERPLANE SERIES PROPERTY}

In this section we are going to provide many classes of Banach spaces $X$ such that $\left(X, X^{*}\right)$ has the AHSP, and so by Theorem 3.6 the pair $\left(\ell_{1}, X\right)$ has the BPBP for bilinear forms. We begin with the class of uniformly smooth Banach spaces. We recall that a Banach space is uniformly smooth if its norm is uniformly Fréchet differentiable at the points of the unit sphere.

Proposition 4.1. If $X$ is a uniformly smooth Banach space, then the pair $\left(X, X^{*}\right)$ has the Approximate Hyperplane Series property.

Proof. If $X$ is uniformly smooth, then $X^{*}$ is uniformly convex (see for instance [14, Theorem 5.5.12]). We will check that the assumption of Proposition 3.3 is satisfied.

Given $\varepsilon>0$, we take $\delta:=2 \delta_{X^{*}}(\varepsilon)$, where $\delta_{X^{*}}(\varepsilon)$ is the modulus of convexity of $X^{*}$, which is a positive real number. Now assume that $x^{*} \in S_{X^{*}}$ and $x_{0} \in S_{X}$ satisfy $\operatorname{Re} x^{*}\left(x_{0}\right)>1-\delta=1-2 \delta_{X^{*}}(\varepsilon)$. If $x_{0}^{*} \in S_{X^{*}}$ satisfies $x_{0}^{*}\left(x_{0}\right)=1$, then

$$
1-\delta_{X^{*}}(\varepsilon)<\operatorname{Re} \frac{x^{*}+x_{0}^{*}}{2}\left(x_{0}\right) \leq \frac{\left\|x^{*}+x_{0}^{*}\right\|}{2} .
$$

Hence $\left\|x^{*}-x_{0}^{*}\right\|<\varepsilon$. By applying Proposition 3.3 we obtain that the pair $\left(X, X^{*}\right)$ has the AHSP.

Let us recall that a Banach space $X$ is smooth if $D(x)$ is a singleton for every $x \in S_{X}$. Not every finite-dimensional space is smooth. However, we will show that every finite-dimensional normed space $X$ also satisfies that $\left(X, X^{*}\right)$ has the AHSP. This fact is a consequence of a refinement of [1, Lemma 3.4] that we will now show. We include the proof of this result for the sake of completeness.

Proposition 4.2. Every finite-dimensional normed space $X$ satisfies that $\left(X, X^{*}\right)$ has the Approximate Hyperplane Series property.

Proof. We are going to check that the hypotheses of Corollary 3.4 hold. We argue by contradiction. Assume that there is some positive real number $\varepsilon_{0}$ not satisfying those hypotheses. Thus, for every positive $\delta>0$ there exists $x_{\delta} \in S_{X}$ satisfying the following condition:

$$
\begin{gathered}
y \in S_{X},\left\|y-x_{\delta}\right\|<\varepsilon_{0} \Rightarrow \\
\exists x^{*} \in S_{X^{*}}: \operatorname{Re} x^{*}\left(x_{\delta}\right)>1-\delta \text { and } \operatorname{dist}\left(x^{*}, D(y)\right) \geq \varepsilon_{0} .
\end{gathered}
$$

Hence, for every $n \in \mathbb{N}$, there is an element $x_{n} \in S_{X}$ satisfying (4.1) for $\delta=\frac{1}{n}$. Since $\operatorname{dim} X<\infty$ we can also assume that $\left(x_{n}\right) \rightarrow x \in S_{X}$ and $\left\|x-x_{n}\right\|<\varepsilon_{0}$ for every natural number $n$.

So by using (4.1), for every $n$ there is $x_{n}^{*} \in S_{X^{*}}$ satisfying

$$
\operatorname{Re} x_{n}^{*}\left(x_{n}\right)>1-\frac{1}{n} \text { and } \operatorname{dist}\left(x_{n}^{*}, D(x)\right) \geq \varepsilon_{0} .
$$

By passing to a subsequence, if needed, we can assume that $\left(x_{n}^{*}\right) \rightarrow x^{*} \in S_{X^{*}}$. In view of (4.2) we have that

$$
x^{*}(x)=1 \text { and } \operatorname{dist}\left(x^{*}, D(x)\right) \geq \varepsilon_{0},
$$

which is a contradiction.

Now we will briefly discuss the assumptions in Corollary 3.4 
Remark 4.3. If $X$ is a smooth Banach space and satisfies the assumptions in Corollary 3.4 then $X^{*}$ is uniformly convex (equivalently, $X$ is uniformly smooth). Furthermore, if $X$ is smooth and $\left(X, X^{*}\right)$ has the Approximate Hyperplane Series property, then $X^{*}$ is uniformly convex.

Proof. We will prove the first statement. The same argument also proves the stronger assertion.

By assumption, for every $\varepsilon>0$ there is $\delta$ satisfying the hypotheses in Corollary 3.4. Take $u^{*}, v^{*} \in S_{X^{*}}$ such that $\left\|u^{*}+v^{*}\right\|>2-\delta$. Hence there is $x \in S_{X}$ satisfying that $\operatorname{Re}\left(u^{*}+v^{*}\right)(x)>2-\delta$, and so we have that

$$
\operatorname{Re} u^{*}(x)>1-\delta, \quad \operatorname{Re} v^{*}(x)>1-\delta .
$$

By assumption, there is $y \in S_{X}$ such that dist $\left(u^{*}, D(y)\right)<\varepsilon$ and dist $\left(v^{*}, D(y)\right)<$ $\varepsilon$. Since $X$ is smooth, then $D(y)=\left\{y^{*}\right\}$, and so we have that $\left\|u^{*}-y^{*}\right\|<\varepsilon$ and $\left\|v^{*}-y^{*}\right\|<\varepsilon$. Finally,

$$
\left\|u^{*}-v^{*}\right\| \leq\left\|u^{*}-y^{*}\right\|+\left\|y^{*}-v^{*}\right\|<2 \varepsilon .
$$

Hence $X^{*}$ is uniformly convex.

Now we will provide an important class of classical Banach spaces that are very far from the uniformly smooth spaces but still satisfy the fact that there is a version of the Bishop-Phelps-Bollobás Theorem for bilinear forms on the product of $\ell_{1}$ and any space in this class.

Proposition 4.4. For every locally compact Hausdorff topological space $\Omega$, the space $Y=\mathcal{C}_{0}(\Omega)$, of real or complex-valued and continuous functions on $\Omega$ vanishing at infinity, satisfies that $\left(Y, Y^{*}\right)$ has the Approximate Hyperplane Series property.

Proof. We prove both the real and the complex cases. It suffices to check that $\mathcal{C}_{0}(\Omega)$ satisfies the assumptions of Proposition 3.3. In order to do that we will use the Riesz Theorem to identify the topological dual of $\mathcal{C}_{0}(\Omega)$ with the space $\mathcal{M}(\Omega)$ of real or complex Radon measures on $\Omega$, endowed with the norm given by the total variation, i.e. given $x^{*} \in \mathcal{C}_{0}(\Omega)^{*}$ there exists a unique $\mu \in \mathcal{M}(\Omega)$, such that

$$
x^{*}(f)=\int_{\Omega} f d \mu, \quad \text { for all } f \in \mathcal{C}_{0}(\Omega)
$$

and $\left\|x^{*}\right\|=|\mu|(\Omega)$, where $|\mu|$ denotes the positive measure called the total variation of $\mu$ (see e.g. [16, 6.19 Theorem]). It is well known that $|\mu|$ is a finite positive regular measure on $\Omega$ and there exists a Borel measurable function $h: \Omega \rightarrow \mathbb{C}$ with $|h(t)|=1$ for all $t$ in $\Omega$ so that

$$
x^{*}(f)=\int_{\Omega} f h d|\mu|, \quad \text { for all } f \in \mathcal{C}_{0}(\Omega)
$$

(see e.g. [16, 6.12 Theorem]). Given $0<\varepsilon<1$, we choose $0<\eta<1$ such that $2 \eta+\sqrt{2 \eta}<\varepsilon$. Let $\left\{y_{j}^{*}: j \in F\right\} \subset S_{\mathcal{C}_{0}(\Omega)^{*}}$ be a finite set and $f_{0} \in S_{\mathcal{C}_{0}(\Omega)}$ such that Re $y_{j}^{*}\left(f_{0}\right)>1-\eta^{2}$, for every $j \in F$. Let $\left\{\mu_{j}: j \in F\right\} \subset S_{\mathcal{M}(\Omega)}$ be such that

$$
y_{j}^{*}(f)=\int_{\Omega} f d \mu_{j}, \quad \text { for all } f \in \mathcal{C}_{0}(\Omega) \text { and all } j \in F .
$$

Let $\left\{h_{j}: j \in F\right\}$ be the Borel measurable functions on $\Omega$ such that

$$
\left|h_{j}\right|=1 \text { and } y_{j}^{*}(f)=\int_{\Omega} f h_{j} d\left|\mu_{j}\right|, \text { for all } j \in F \text { and all } f \in \mathcal{C}_{0}(\Omega) .
$$


We are assuming that

$$
\int_{\Omega} \operatorname{Re}\left(f_{0} h_{j}\right) d\left|\mu_{j}\right|=\operatorname{Re}\left(\int_{\Omega} f_{0} h_{j} d\left|\mu_{j}\right|\right)=\operatorname{Re} y_{j}^{*}\left(f_{0}\right)>1-\eta^{2}, \text { for all } j \in F .
$$

Now for each $j \in F$ let us consider the Borel set

$$
B_{j}=\left\{t \in \Omega: \operatorname{Re}\left(f_{0}(t) h_{j}(t)\right)>1-\eta\right\} .
$$

For every $j \in F$ we also have

$$
\begin{gathered}
1-\eta^{2}<\int_{\Omega} \operatorname{Re}\left(f_{0} h_{j}\right) d\left|\mu_{j}\right|=\int_{B_{j}} \operatorname{Re}\left(f_{0} h_{j}\right) d\left|\mu_{j}\right|+\int_{\Omega \backslash B_{j}} \operatorname{Re}\left(f_{0} h_{j}\right) d\left|\mu_{j}\right| \\
\leq \int_{B_{j}} d\left|\mu_{j}\right|+(1-\eta) \int_{\Omega \backslash B_{j}} d\left|\mu_{j}\right| \\
=\left|\mu_{j}\right|\left(B_{j}\right)+(1-\eta)\left|\mu_{j}\right|\left(\Omega \backslash B_{j}\right)=\eta\left|\mu_{j}\right|\left(B_{j}\right)+1-\eta .
\end{gathered}
$$

Hence

$$
\left|\mu_{j}\right|\left(B_{j}\right)>1-\eta \text {. }
$$

Since each $\left|\mu_{j}\right|$ is regular, then for each $j \in F$ there is a compact set $K_{j} \subset B_{j}$, such that

$$
\left|\mu_{j}\right|\left(K_{j}\right)>1-\eta>0, \text { for all } j \in F \text {. }
$$

As a consequence,

$$
\left|\mu_{j}\right|\left(\Omega \backslash K_{j}\right)<\eta, \text { for every } j \in F .
$$

Let us take $K:=\bigcup_{j \in F} K_{j}$, which is a compact subset of $\Omega$ satisfying

$$
K=\bigcup_{j \in F} K_{j} \subset \bigcup_{j \in F} B_{j} \subset\left\{t \in \Omega:\left|f_{0}(t)\right|>1-\eta\right\} .
$$

The set $U:=\left\{t \in \Omega:\left|f_{0}(t)\right|>1-\eta\right\}$ is open. Since every locally compact space is completely regular, there is a function $m \in \mathcal{C}_{0}(\Omega)$ that separates the closed set $\Omega \backslash U$ and the compact set $K$, i.e., such that $0 \leq m \leq 1, m(K)=\{1\}$ and $m(\Omega \backslash U)=\{0\}$. So the function $h_{0}$ defined on $\Omega$ by

$$
h_{0}(t):= \begin{cases}\frac{f_{0}(t)}{\left|f_{0}(t)\right|} m(t) & \text { if } t \in U, \\ 0 & \text { if } t \in \Omega \backslash U\end{cases}
$$

is continuous, and since it vanishes outside the relatively compact set $U$, it belongs to $\mathcal{C}_{0}(\Omega)$. We take $g_{0}:=h_{0}+(1-m) f_{0}$, which is also a continuous function on $\Omega$ vanishing at infinity.

It is also clear that $g_{0}$ satisfies

$$
\left|g_{0}(t)\right| \leq m(t)+1-m(t)=1, \text { for all } t \in \Omega
$$

and $\left|g_{0}\right|(K)=\{1\}$, so $g_{0} \in S_{\mathcal{C}_{0}(\Omega)}$.

Now we will check that

$$
\left\|g_{0}-f_{0}\right\| \leq \eta<\varepsilon
$$

If $t \in U$, then $\left|f_{0}(t)\right|>1-\eta$, so

$$
\left|g_{0}(t)-f_{0}(t)\right|=\left|\frac{f_{0}(t)}{\left|f_{0}(t)\right|} m(t)-m(t) f_{0}(t)\right|=m(t)|1-| f_{0}(t)||<\eta .
$$

In the case that $t \in \Omega \backslash U$ we have that $m(t)=0=h_{0}(t)$, so $g_{0}(t)-f_{0}(t)=0$. 
Now we will provide the new set of continuous functionals that satisfy the desired condition. For each $j \in F$ let us define the functional

$$
z_{j}^{*}(f):=\frac{1}{\left|\mu_{j}\right|\left(K_{j}\right)} \int_{K_{j}} f \frac{\overline{f_{0}}}{\left|f_{0}\right|} d\left|\mu_{j}\right| \quad\left(f \in \mathcal{C}_{0}(\Omega)\right) .
$$

Obviously $\left\{z_{j}^{*}: j \in F\right\} \subset S_{\mathcal{C}_{0}(\Omega)^{*}}$, and for every $f \in \mathcal{C}_{0}(\Omega)$ we have

$$
\begin{gathered}
\left|y_{j}^{*}(f)-z_{j}^{*}(f)\right| \leq\left|\int_{\Omega \backslash K_{j}} f h_{j} d\right| \mu_{j}||+\left|\int_{K_{j}}\left(f-f \frac{\overline{f_{0}}}{\left|f_{0}\right|} \bar{h}_{j}\right) h_{j} d\right| \mu_{j}|| \\
+\left(\frac{1}{\left|\mu_{j}\right|\left(K_{j}\right)}-1\right)\left|\int_{K_{j}} f \frac{\overline{f_{0}}}{\left|f_{0}\right|} d\right| \mu_{j}|| \\
\leq\left|\mu_{j}\right|\left(\Omega \backslash K_{j}\right)\|f\|+\int_{K_{j}}\left|\left(1-\frac{\overline{f_{0}}}{\left|f_{0}\right|} \bar{h}_{j}\right) f\right| d\left|\mu_{j}\right|+\left(\frac{1}{\left|\mu_{j}\right|\left(K_{j}\right)}-1\right)\left|\mu_{j}\right|\left(K_{j}\right)\|f\| \\
\left.\leq \eta\|f\|+\int_{K_{j}} \sqrt{2 \eta}\|f\| d\left|\mu_{j}\right|+\left(1-\left|\mu_{j}\right|\left(K_{j}\right)\right)\|f\| \quad \text { (by (4.4) and Lemma 3.5) }\right) \\
\leq(\eta+\sqrt{2 \eta}+\eta)\|f\|=(2 \eta+\sqrt{2 \eta})\|f\| \quad \text { (by (4.3) }) .
\end{gathered}
$$

Hence

$$
\left\|y_{j}^{*}-z_{j}^{*}\right\| \leq 2 \eta+\sqrt{2 \eta}<\varepsilon, \text { for all } j \in F
$$

Finally, for each $j \in F$ we have that

$$
z_{j}^{*}\left(g_{0}\right)=\frac{1}{\left|\mu_{j}\right|\left(K_{j}\right)} \int_{K_{j}} g_{0} \frac{\overline{f_{0}}}{\left|f_{0}\right|} d\left|\mu_{j}\right|=\frac{1}{\left|\mu_{j}\right|\left(K_{j}\right)} \int_{K_{j}} \frac{f_{0}}{\left|f_{0}\right|} \frac{\overline{f_{0}}}{\left|f_{0}\right|} d\left|\mu_{j}\right|=1 .
$$

In view of (4.5), (4.6) and the last equality, the proof is completed.

Corollary 4.5. For every Hausdorff and compact topological space $K$, the space $Y=\mathcal{C}(K)$, of real or complex-valued and continuous functions on $K$, satisfies that $\left(Y, Y^{*}\right)$ has the Approximate Hyperplane Series property.

Corollary 4.6. If $X=c_{0}$ (real or complex case), then $\left(X, X^{*}\right)$ has the Approximate Hyperplane Series property.

Proposition 4.7. If $H$ is a Hilbert space and $X=K(H)$, the space of compact operators on $H$, then $\left(X, X^{*}\right)$ has the Approximate Hyperplane Series property.

Proof. In the finite-dimensional case it suffices to use Proposition 4.2, So we can assume that $H$ is infinite-dimensional.

We will use the standard identification of $K(H)^{*}$ and the space of nuclear operators on $H$, endowed with the nuclear norm (see for instance [18, Theorem 1, p. 46], [17, Theorem 5.6] or [11, Theorem 16.50]). Indeed we will prove that the assumptions of Corollary 3.4 are satisfied.

Given $\varepsilon>0$, we choose $0<\eta<\frac{1}{2}$ such that

$$
2 \sqrt{2 \eta}+5 \eta<\varepsilon
$$

Let us fix any element $S_{0} \in S_{K(H)}$. By using the polar decomposition of $S_{0}$ (see for instance [17, Lemma 5.5]), there are orthonormal systems $\left(y_{n}\right)$ and $\left(x_{k}\right)$ in $H$ such that

$$
S_{0}:=\sum_{n=1}^{\infty} a_{k} y_{k}^{*} \otimes x_{k}
$$


where $\left(a_{k}\right)$ is a decreasing sequence of real numbers convergent to zero, $1=\left\|S_{0}\right\|=$ $\max \left\{a_{n}: n \in \mathbb{N}\right\}$ and $y_{k}^{*}(x)=\left(x \mid y_{k}\right)$ for each $k \in \mathbb{N}$ and $x \in H$, where $(\cdot \mid \cdot)$ denotes the inner product of $H$. Let us take $s:=1-\eta$ and

$$
B:=\left\{n \in \mathbb{N}: a_{n}>s\right\} .
$$

It is clear that $B$ is finite and non-empty. So the operator $T_{0}$ on $H$, defined as

$$
T_{0}:=\sum_{n \in B} y_{k}^{*} \otimes x_{k}+\sum_{n \in \mathbb{N} \backslash B} a_{k} y_{k}^{*} \otimes x_{k},
$$

belongs to $S_{K(H)}$ and satisfies

$$
\left\|T_{0}-S_{0}\right\|=\max \left\{1-a_{n}: n \in B\right\}<1-s=\eta<\varepsilon .
$$

Now take $t:=1-\eta^{4}$ and choose any element $z^{*} \in S_{K(H)^{*}}$ such that $\operatorname{Re} z^{*}\left(S_{0}\right)>$ $t$. By the description of $K(H)^{*}$ there are a sequence $\left(b_{n}\right) \in \ell_{1}$ of nonnegative real numbers, and orthonormal systems $\left(f_{n}\right)$ and $\left(e_{n}\right)$ in $H$ such that

$$
z^{*}(T)=\sum_{n=1}^{\infty} b_{n}\left(T\left(e_{n}\right) \mid f_{n}\right), \text { for all } T \in K(H) .
$$

Also, it is satisfied that $1=\left\|z^{*}\right\|=\sum_{n=1}^{\infty} b_{n}$ (see for instance [18, Theorem 5, p. 42] or [17, Lemma 5.41]). In such a case we will write $z^{*}=\sum_{n=1}^{\infty} b_{n} f_{n}^{*} \otimes e_{n}$. We have

$$
1-\eta^{4}=t<\operatorname{Re} z^{*}\left(S_{0}\right)=\operatorname{Re} \sum_{n=1}^{\infty} b_{n}\left(S_{0}\left(e_{n}\right) \mid f_{n}\right) .
$$

If we consider the set $C$ given by $C:=\left\{n \in \mathbb{N}: \operatorname{Re}\left(S_{0}\left(e_{n}\right) \mid f_{n}\right) \geq 1-\eta^{3}\right\}$, then Lemma 3.2 with $r:=1-\eta^{3}$ gives

$$
\sum_{n \in C} b_{n}>1-\eta>0, \text { and so } \sum_{n \in \mathbb{N} \backslash C} b_{n}<\eta .
$$

Now we define the element $y^{*} \in K(H)^{*}$ given by

$$
y^{*}=\frac{1}{\sum_{n \in C} b_{n}} \sum_{n \in C} b_{n} f_{n}^{*} \otimes e_{n} .
$$

Then $\left\|y^{*}\right\| \leq 1$, and in view of (4.8) we have that

$$
\begin{aligned}
\left\|y^{*}-z^{*}\right\| & =\left\|\frac{1}{\sum_{n \in C} b_{n}} \sum_{n \in C} b_{n} f_{n}^{*} \otimes e_{n}-\sum_{n=1}^{\infty} b_{n} f_{n}^{*} \otimes e_{n}\right\| \\
& \leq\left(\frac{1}{\sum_{n \in C} b_{n}}-1\right)\left\|\sum_{n \in C} b_{n} f_{n}^{*} \otimes e_{n}\right\|+\left\|\sum_{n \in \mathbb{N} \backslash C} b_{n} f_{n}^{*} \otimes e_{n}\right\| \\
& \leq 2\left(1-\sum_{n \in C} b_{n}\right)<2 \eta .
\end{aligned}
$$

Now for each $n \in C$ we will use the parallelogram law for the elements $S_{0}\left(e_{n}\right)$ and $f_{n}$ and obtain that

$$
\begin{gathered}
r^{2}+1+2 r+\left\|S_{0}\left(e_{n}\right)-f_{n}\right\|^{2} \\
\leq\left(\operatorname{Re}\left(\left(S_{0}\left(e_{n}\right)+f_{n}\right) \mid f_{n}\right)\right)^{2}+\left\|S_{0}\left(e_{n}\right)-f_{n}\right\|^{2} \\
\leq\left\|S_{0}\left(e_{n}\right)+f_{n}\right\|^{2}+\left\|S_{0}\left(e_{n}\right)-f_{n}\right\|^{2} \\
=2\left(\left\|S_{0}\left(e_{n}\right)\right\|^{2}+\left\|f_{n}\right\|^{2}\right) \leq 4 .
\end{gathered}
$$


We deduce that

$$
\left\|S_{0}\left(e_{n}\right)-f_{n}\right\|^{2} \leq 4-\left(r^{2}+2 r+1\right)<4 \eta^{3}, \text { for all } n \in C .
$$

Now by using (4.7) we deduce

$$
\begin{gathered}
\left\|T_{0}\left(e_{n}\right)-f_{n}\right\| \leq\left\|T_{0}\left(e_{n}\right)-S_{0}\left(e_{n}\right)\right\|+\left\|S_{0}\left(e_{n}\right)-f_{n}\right\| \\
\leq\left\|T_{0}-S_{0}\right\|+2 \eta \leq 3 \eta, \quad \forall n \in C .
\end{gathered}
$$

We also know that $\left\|S_{0}\left(e_{n}\right)\right\| \geq r$ for every $n \in C$. Let us denote by $P$ the orthogonal projection on $H$ onto the subspace $Y$ generated by $\left\{y_{k}: k \in B\right\}$. For each $n \in C$ we have that

$$
\begin{gathered}
r^{2} \leq\left\|S_{0}\left(e_{n}\right)\right\|^{2} \\
=\left\|\sum_{k \in B} a_{k}\left(e_{n} \mid y_{k}\right) x_{k}+\sum_{k \in \mathbb{N} \backslash B} a_{k}\left(e_{n} \mid y_{k}\right) x_{k}\right\|^{2} \\
\leq \sum_{k \in B}\left|a_{k}\right|^{2}\left|\left(P\left(e_{n}\right) \mid y_{k}\right)\right|^{2}+\sum_{k \in \mathbb{N} \backslash B}\left|a_{k}\right|^{2}\left|\left(e_{n}-P\left(e_{n}\right) \mid y_{k}\right)\right|^{2} \\
\leq\left\|P\left(e_{n}\right)\right\|^{2}+s^{2}\left\|e_{n}-P\left(e_{n}\right)\right\|^{2} \\
=\left\|P\left(e_{n}\right)\right\|^{2}+s^{2}\left(1-\left\|P\left(e_{n}\right)\right\|^{2}\right)=\left\|P\left(e_{n}\right)\right\|^{2}\left(1-s^{2}\right)+s^{2} .
\end{gathered}
$$

Hence

$$
\left\|P\left(e_{n}\right)\right\|^{2} \geq \frac{r^{2}-s^{2}}{1-s^{2}}=1-\frac{\eta^{2}\left(2-\eta^{3}\right)}{2-\eta}>1-\eta>0
$$

that is,

$$
\left\|e_{n}-P\left(e_{n}\right)\right\|^{2}<\eta
$$

As a consequence

$$
\begin{gathered}
\left\|e_{n}-\frac{P\left(e_{n}\right)}{\left\|P\left(e_{n}\right)\right\|}\right\|^{2}=\left\|P\left(e_{n}\right)-\frac{P\left(e_{n}\right)}{\left\|P\left(e_{n}\right)\right\|}\right\|^{2}+\left\|e_{n}-P\left(e_{n}\right)\right\|^{2} \\
=\left|1-\left\|P\left(e_{n}\right)\right\|\right|^{2}+\left\|e_{n}-P\left(e_{n}\right)\right\|^{2} \leq 2 \eta .
\end{gathered}
$$

We just checked that

$$
\left\|e_{n}-\frac{P\left(e_{n}\right)}{\left\|P\left(e_{n}\right)\right\|}\right\| \leq \sqrt{2 \eta} .
$$

Let us denote $\tilde{e}_{n}:=\frac{P\left(e_{n}\right)}{\left\|P\left(e_{n}\right)\right\|}$ for each $n \in C$. Then we know that

$$
\tilde{e}_{n} \in S_{H} \cap Y \quad \text { and } \quad\left\|\tilde{e}_{n}-e_{n}\right\| \leq \sqrt{2 \eta}, \text { for all } n \in C .
$$

Finally we take the functional $x^{*} \in K(H)^{*}$ given by

$$
x^{*}=\frac{1}{\sum_{n \in C} b_{n}} \sum_{n \in C} b_{n} T_{0}\left(\tilde{e}_{n}\right)^{*} \otimes \tilde{e}_{n} .
$$

It is clear that $x^{*} \in B_{K(H)^{*}}$. It is also clear that $\left\|T_{0}\left(\tilde{e}_{n}\right)\right\|=1$ for every $n \in C$, and so

(4.12) $x^{*}\left(T_{0}\right)=\frac{1}{\sum_{n \in C} b_{n}} \sum_{n \in C} b_{n}\left(T_{0}\left(\tilde{e}_{n}\right) \mid T_{0}\left(\tilde{e}_{n}\right)\right)=1, \quad$ hence $\quad x^{*} \in S_{K(H)^{*}}$. 
Now we estimate

$$
\begin{gathered}
\sum_{n \in C} b_{n}\left\|x^{*}-y^{*}\right\|=\left\|\sum_{n \in C} b_{n} T_{0}\left(\tilde{e}_{n}\right)^{*} \otimes \tilde{e}_{n}-\sum_{n \in C} b_{n} f_{n}^{*} \otimes e_{n}\right\| \\
\leq\left\|\sum_{n \in C} b_{n} T_{0}\left(\tilde{e}_{n}\right)^{*} \otimes \tilde{e}_{n}-\sum_{n \in C} b_{n} T_{0}\left(\tilde{e}_{n}\right)^{*} \otimes e_{n}\right\| \\
+\left\|\sum_{n \in C} b_{n} T_{0}\left(\tilde{e}_{n}\right)^{*} \otimes e_{n}-\sum_{n \in C} b_{n} T_{0}\left(e_{n}\right)^{*} \otimes e_{n}\right\| \\
\quad+\left\|\sum_{n \in C} b_{n} T_{0}\left(e_{n}\right)^{*} \otimes e_{n}-\sum_{n \in C} b_{n} f_{n}^{*} \otimes e_{n}\right\| \\
\leq 2 \sum_{n \in C} b_{n}\left\|\tilde{e}_{n}-e_{n}\right\|+\sum_{n \in C} b_{n}\left\|T_{0}\left(e_{n}\right)-f_{n}\right\| \\
\leq \sum_{n \in C} b_{n}(2 \sqrt{2 \eta}+3 \eta) \quad(\text { by (4.11) and (4.10) }) .
\end{gathered}
$$

We have obtained that

$$
\left\|x^{*}-y^{*}\right\| \leq 2 \sqrt{2 \eta}+3 \eta .
$$

Finally, in view of (4.9) we have that

$$
\begin{gathered}
\left\|x^{*}-z^{*}\right\| \leq\left\|x^{*}-y^{*}\right\|+\left\|y^{*}-z^{*}\right\| \\
\leq 2 \sqrt{2 \eta}+5 \eta<\varepsilon .
\end{gathered}
$$

By (4.7), 4.12) and the previous inequality, we can apply Corollary 3.4 and this completes the proof.

Choi and Song 9 proved that there is no version of the Bishop-Phelps-Bollobás Theorem for bilinear forms. Indeed it happens for $\ell_{1}$. Hence, by Theorem 3.6 the pair $\left(\ell_{1}, \ell_{1}^{*}\right)$ does not have the AHSP. Actually we will show that for every infinite-dimensional $L_{1}(\mu)$ the pair $\left(L_{1}(\mu), L_{1}(\mu)^{*}\right)$ fails the AHSP.

Proposition 4.8. For every infinite-dimensional space $L_{1}(\mu)$, the pair $\left(L_{1}(\mu)\right.$, $\left.L_{1}(\mu)^{*}\right)$ fails the Approximate Hyperplane Series property.

Proof. Since $L_{1}(\mu)$ is infinite-dimensional, there exists a sequence $\left(A_{n}\right)_{n=1}^{\infty}$ of pairwise disjoint measurable sets with $0<\mu\left(A_{n}\right)<\infty$ for every $n \in \mathbb{N}$. Assume that the pair $\left(L_{1}(\mu), L_{1}(\mu)^{*}\right)$ has the AHSP. Given $0<\varepsilon<\frac{1}{2}$, let $0<\delta, \eta<\varepsilon$ be the positive real numbers satisfying the conditions in Definition 3.1 Let us choose $n \in \mathbb{N}, n \geq 2$ such that $\frac{1}{n}<\min \{\delta, \eta\}$ and take

$$
f_{0}=\frac{1}{n} \sum_{j=1}^{n} \frac{1}{\mu\left(A_{j}\right)} \chi_{A_{j}} \quad \text { and } \quad g_{i}=\sum_{j=1, j \neq i}^{n} \chi_{A_{j}},
$$

for $1 \leq i \leq n$. It is clear that $f_{0} \in S_{L_{1}(\mu)}$ and $g_{i} \in L_{\infty}(\mu)$ with $\left\|g_{i}\right\|_{\infty}=1$ for every $i$, where $\|\cdot\|_{\infty}$ denotes the usual norm in $L_{\infty}(\mu)$. Since $0<\mu\left(A_{j}\right)<\infty$ for every $j, g_{i}$ is associated to an element in the unit sphere of $L_{1}(\mu)^{*}$. We will denote by $x_{i}^{*}$ the element in $L_{1}(\mu)^{*}$ corresponding to $g_{i}$ for $1 \leq i \leq n$. It is satisfied that

$$
x_{i}^{*}\left(f_{0}\right)=\int_{\Omega} g_{i} f_{0} d \mu=\frac{1}{n} \sum_{j=1, j \neq i}^{n} \frac{1}{\mu\left(A_{j}\right)} \int_{\Omega} \chi_{A_{j}} d \mu=\frac{n-1}{n},
$$


for $i=1, \ldots, n$. Thus, the convex series $\sum_{i=1}^{n} \frac{1}{n} x_{i}^{*}$ satisfies

$$
\left(\sum_{i=1}^{n} \frac{1}{n} x_{i}^{*}\right)\left(f_{0}\right)=\frac{n-1}{n}>1-\delta .
$$

Since we are assuming that the AHSP is satisfied, there exist $C \subset\{1, \ldots, n\}$ and $\left\{y_{k}^{*}: k \in C\right\} \subset S_{L_{1}(\mu)^{*}}$ and $f \in S_{L_{1}(\mu)}$ such that

$\frac{\operatorname{card}(C)}{n}>1-\delta, \quad\left\|y_{k}^{*}-x_{k}^{*}\right\|<\varepsilon, \quad y_{k}^{*}(f)=1$, for all $k \in C$, and $\left\|f-f_{0}\right\|_{1}<\varepsilon$,

where $\|\cdot\|_{1}$ denotes the usual norm in $L_{1}(\mu)$. Now let us notice that for every function $h \in S_{L_{\infty}(\mu)}$ satisfying $\left|\int_{\Omega} f h d \mu\right|=1$, if for some measurable set $A$ we have $\left\|h \chi_{A}\right\|_{\infty}<1$, since

$$
1=\left|\int_{\Omega} f h d \mu\right| \leq \int_{A}\left\|h \chi_{A}\right\|_{\infty}|f| d \mu+\int_{\Omega \backslash A}|f| d \mu=1+\left(\left\|h \chi_{A}\right\|_{\infty}-1\right) \int_{\Omega}|f| \chi_{A} d \mu,
$$

then it follows that $|f| \chi_{A}=0$ almost everywhere. In our case, since the support of $f$ is a countable union of measurable sets of finite measure and every $A_{i}$ has finite measure, if we take $B:=\operatorname{supp} f \cup \bigcup_{j=1}^{n} A_{j}$, the restriction of $y_{k}^{*}$ to $L_{1}\left(\mu_{\mid B}\right)$ is represented by a function $h_{k} \in L_{\infty}(\mu)$ for every $k \in C$. Hence we have that

$$
y_{k}^{*}(f)=\int_{\Omega} f h_{k} d \mu=1, \quad\left\|h_{k}-g_{k}\right\|_{\infty} \leq\left\|y_{k}^{*}-x_{k}^{*}\right\|<\varepsilon, \text { for all } k \in C,
$$

and so $\left\|h_{k} \chi_{A_{k}}\right\|_{\infty}<\varepsilon$ for $k \in C$. Thus $f \chi_{A_{k}}=0$ a.e. for every $k \in C$. As a consequence

$$
\left\|f-f_{0}\right\|_{1} \geq \int_{\bigcup_{k \in C} A_{k}}\left|f_{0}\right| d \mu=\frac{\operatorname{card}(C)}{n}>1-\delta>\frac{1}{2}>\varepsilon,
$$

which is a contradiction.

\section{ACKNOWLEDGMENT}

It is the authors' pleasure to thank Vladimir Kadets and Rafael Payá for some useful comments.

\section{REFERENCES}

[1] M.D. Acosta, R.M. Aron, D. García and M. Maestre, The Bishop-Phelps-Bollobás theorem for operators, J. Funct. Anal. 254 (11) (2008), 2780-2799. MR2414220 (2009c:46016)

[2] R.M. Aron, B. Cascales and O. Kozhushkina, The Bishop-Phelps-Bollobás Theorem and Asplund operators, Proc. Amer. Math. Soc. 139 (2011), 3553-3560. MR2813386 (2012d:46046)

[3] R.M. Aron, Y.S. Choi, D. García and M. Maestre, The Bishop-Phelps-Bollobás theorem for $\mathcal{L}\left(L_{1}(\mu), L_{\infty}[0,1]\right)$, Adv. Math. 228 (2011), 617-628. MR 2822241 (2012f:46023)

[4] E. Bishop and R.R. Phelps, A proof that every Banach space is subreflexive, Bull. Amer. Math. Soc. (N.S.) 67 (1961), 97-98. MR0123174 (23:A503)

[5] B. Bollobás, An extension to the theorem of Bishop and Phelps, Bull. London Math. Soc. 2 (1970), 181-182. MR0267380(42:2282)

[6] F.F. Bonsall and J. Duncan, Numerical Ranges II, Lecture Note Series 10 London Math. Soc., Cambridge University Press, Cambridge, 1973. MR 0442682 (56:1063)

[7] L. Cheng, D. Dai and Y. Dong, A sharp operator version of the Bishop-Phelps Theorem for operators from $\ell_{1}$ to $C L$-spaces, Proc. Amer. Math. Soc. 141 (2013), 867-872. MR3003679

[8] Y.S. Choi and S.K. Kim, The Bishop-Phelps-Bollobás property and lush space, J. Math. Anal. Appl. 390 (2012), 549-555. MR2890536 
[9] Y.S. Choi and H.G. Song, The Bishop-Phelps-Bollobás theorem fails for bilinear forms on $\ell_{1} \times \ell_{1}$, J. Math. Anal. Appl. 360 (2009), 752-753. MR2561271 (2010h:46016)

[10] D. Dai, The Bishop-Phelps-Bollobás Theorem for bilinear mappings, preprint, 2011.

[11] M. Fabian, P. Habala, P. Hájek, V. Montesinos and V. Zizler, Banach space Theory. The basis for linear and nonlinear analysis, CMS Books in Mathematics/Ouvrages de Mathématiques de la SMC, Springer-Verlgag, New York, 2011. MR2766381

[12] C. Franchetti and R. Payá, Banach spaces with strongly differentiable norm, Boll. U.M.I. 7 (1993), 45-70. MR 1216708 (94d:46015)

[13] M. Martín and R. Payá, On CL-spaces and almost CL-spaces, Ark. Mat. 42 (2004), 107-118. MR2056547(2005e:46019)

[14] R.E. Megginson, An introduction to Banach space theory, Graduate Texts in Mathematics 183, Springer-Verlag, New York, 1998. MR.1650235 (99k:46002)

[15] R. Payá and Y. Saleh, New sufficient conditions for the denseness of norm attaining multilinear forms, Bull. London Math. Soc. 34 (2002), 212-218. MR1874249 (2003a:46033)

[16] W. Rudin, Real and complex analysis, McGraw-Hill Book Co., London, 1970.

[17] R. Schatten, A Theory of cross spaces, Ann. of Math. Studies no. 26, Princeton Univ. Press, Princeton, N.J., 1950. MR0036935 (12:186e)

[18] R. Schatten, Norm ideals of completely continuous operators, Springer-Verlag, BerlinGöttingen-Heidelberg, 1960. MR0119112 (22:9878)

[19] C. Stegall, Optimization and differentiation in Banach spaces, Linear Algebra Appl. 84 (1986), 191-211. MR872283 (88a:49005)

Departamento de Análisis Matemático, Universidad de Granada, Facultad de CienCIAS, 18071-Granada, Spain

E-mail address: dacosta@ugr.es

Departamento de Análisis Matemático, Universidad de Granada, Facultad de CienCIAS, 18071-GranadA, SpAIN

E-mail address: juliobg@ugr.es

Departamento de Análisis Matemático, Universidad de Valencia, Doctor Moliner 50, 46100 Burjasot Valencia, Spain

E-mail address: domingo.garcia@uv.es

Departamento de Análisis Matemático, Universidad de Valencia, Doctor Moliner 50, 46100 Burjasot Valencia, Spain

E-mail address: manuel.maestre@uv.es 